\title{
Computation Rate Maximization in Wireless Powered MEC with Spread Spectrum Multiple Access
}

\author{
Yuegui Chen, Suzhi Bi, Xian Li, Xiaohui Lin, and Hui Wang \\ College of Electronic and Information Engineering, Shenzhen University, Shenzhen, China. \\ E-mail: chenyuegui2017@email.szu.edu.cn, \{bsz, xianli, xhlin,wanghsz\}@szu.edu.cn.
}

\begin{abstract}
The integration of mobile edge computing (MEC) and wireless power transfer (WPT) technologies has recently emerged as an effective solution for extending battery life and increasing the computing power of wireless devices. In this paper, we study the resource allocation problem of a multi-user wireless powered MEC system, where the users share the wireless channel via direct sequence code division multiple access (DSCDMA). In particular, we are interested in jointly optimizing the task offloading decisions and resource allocation, to maximize the weighted sum computation rate of all the users in the network. The optimization problem is formulated as a mixed integer non-linear programming (MINLP). For a given offloading user set, we implement an efficient Fractional Programming (FP) approach to mitigate the multi-user interference in the uplink task offloading. On top of that, we then propose a Stochastic Local Search algorithm to optimize the offloading decisions. Simulation results show that the proposed method can effectively enhance the computing performance of a wireless powered MEC with spread spectrum multiple access compared to other representative benchmark methods.
\end{abstract}

Index Terms-Mobile edge computing, wireless power transfer, resource allocation, spread spectrum access, integer optimization.

\section{INTRODUCTION}

With the explosive development of the Internet of things (IoT), new applications have emerged with advanced features that require consistent high-performance computing service and low processing latency, such as autonomous driving, smart appliances, virtual reality and remote surgery. New computing performance requirements have also spawned a new computing paradigm named Mobile Edge Computing (MEC), which deploys cost-efficient servers within the radio access network, e.g., at WiFi access points (APs) or the cellular radio network controller site, to reduce the backhaul transmission delay in traditional cloud computing system [1]. There has been extensive studies on optimizing the MEC system computing performance by jointing optimizing task offloading decisions and system resource allocation [2]-[4]. However, the performance of conventional MEC system is limited by the finite battery life of WDs, especially for size-constrained IoT devices. Recently, radio-frequency (RF) signal based wireless power transfer (WPT) provides a viable solution that can charge the battery continuously in distance without interrupting its normal operation [5], [6]. There have been some recent studies on integrating WPT to conventional MEC systems [7]-[9]. For instance, [7] considers a multi-user wireless powered MEC network and maximizes the weighted computation rate of all the users by jointly optimizing the task offloading decisions and the system transmission time allocation. The work is later extended in [8] to use a deep reenforcement learning approach for optimizing the offloading decisions.

The existing studies have considered many multiple access schemes for the task-offloading users to share the common spectrum, such as TDMA (e.g., in [4], [7]), FDMA (e.g., in [10]), OFDMA (e.g., in [11]) and non-orthogonal multiple access (NOMA) (e.g., in [12]). In pracitce, spread spectrum multiple access (such as DS-CDMA) is a standardized multiple access method widely used in IoT systems, such as in LoRaWAN and SigFox [13], due to its simplicity in hosting massive number of simultaneous connections. Nevertheless, to the authors' best knowledge, it lacks relevant research in prior works on applying spread spectrum multiple access to wireless powered MEC. In this paper, we consider a multiuser wirelesspowered MEC system using DS-CDMA to coordinate the task offloading of the users. Our main goal is to maximize the weighted sum of computation rates across all the users. The main contributions of this paper are as follows:

1) We study the computation rate maximization problem in a wireless powered MEC system where the users perform task offloading based on DS-CDMA. The problem is formulated as a mixed integer non-linear programming (MINLP) that jointly optimizes the time allocation on WPT and task offloading, the binary offloading decisions of the WDs, and the transmit power control of the offloading WDs.

2) To tackle the problem, we first consider the case that the set of offloading WDs is given. The remaining optimization on time allocation and multi-user power control, however, is still a hard non-convex problem. For a given WPT time, we adopt a fractional programming(FP) approach to maximize the weighted sum computation rate of the users in the interference channel. Then, the optimal time allocation can be easily obtained through a simple one-dimension search method, e.g., goldensection search.

3) On top of the WPT time allocation and power control method, we further propose an integer Stochastic Local Search method to optimize the combinatorial compu- 
tation offloading decisions. The method is based on iterative update of user binary offloading decision, and shown to converge to a good solution within limited iterations.

\section{SYSTEM MODEL}

\section{A. Network Model}

As shown in Fig. 1, we consider a multiuser wirelesspowered MEC system with $N$ users and one access point (AP) that is integrated with a RF energy transmitter and an MEC server. Each of the device is equipped with a single antenna. The AP broadcasts RF energy while all users harvest energy and charge the battery with the received energy. In this paper, we consider the binary computation offloading policy [1], where the task is indivisible, such that a WD either operates in offloading mode (mode 1, like WD1 and WD2 in Fig. 1) or local computing mode (mode 0, like WD3). Let $\mathcal{N}_{1}$ and $\mathcal{N}_{0}$ denote the two non-overlapping index sets of WDs that operate in mode- 1 and mode- 0 , respectively, where $\mathcal{N}=\mathcal{N}_{1} \cup \mathcal{N}_{0}=\{1, \ldots, N\}$ denotes the set of all the users. To avoid co-channel interference, the downlink WPT and the uplink wireless communication (for offloading) operate in a time-division-multiplexing (TDD) manner. The mode-1 users offload their task data in the uplink to the MEC server simultaneously using CDMA. As shown in Fig.1, within each system time frame of duration $T$, the transmission time is divided into three phases: the first phase $\alpha T$ is used for the AP broadcasting RF energy to the WDs, where $\alpha \in(0,1)$, the second phase is for the mode- 1 users offloading the task data, and the AP computes and returns the computation results back to the users in the last phase. Because of the much stronger computation capability and transmit power of the AP, as well as the relatively short computation result, we neglect the time consumed on task computing and result downloading (as in [7] [10]). Therefore, the second phase occupies $(1-\alpha) T$ amount of time.

During the first period $\alpha T$, HAP broadcasts RF power to all WDs in the downlink with fixed transmit power $P_{0}$, the energy collected by the $i$-th user is expressed as follows 1 :

$$
E_{i}=\nu h_{i} P_{0} \alpha T, i=1,2, \ldots, N,
$$

where $h_{i}$ denotes the channel power gain from the AP to the $i$-th WDs, which is assumed equal for both the uplink and downlink for simplicity. $\nu \in(0,1)$ is the energy harvesting efficiency. After WET, mode-1 users offload their tasks for edge computation for the rest of the time frame.

\section{B. Offloading Mode}

Due to the TDD circuit constraint, a mode-1 user starts offloading its task data for edge execution using DS-CDMA after harvesting RF energy. Suppose that a pseudo-random code of processing gain $G$ is used as the spreading sequence,

\footnotetext{
${ }^{1}$ We consider a linear energy harvesting model in this paper, as it is shown that the non-linear saturation effect of a single energy harvesting circuit can be effectively rectified by using multiple energy harvesting circuits concatenated in parallel, resulting in a sufficiently large linear conversion region in practice 15
}

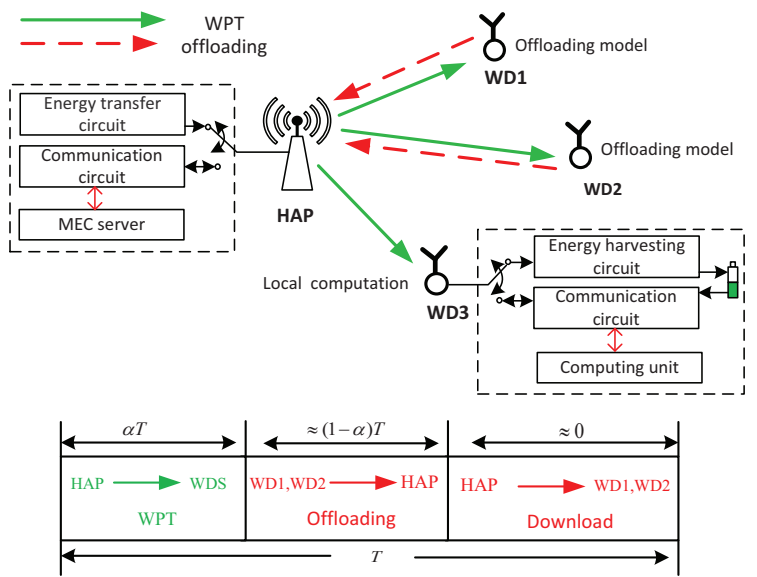

Fig. 1. The model for 3-user WPT-MEC system with binary computation offloading and system time allocation

the maximum amount of information offloaded to the edge by the $i$-th user is [14, Sec. 4]

$$
\begin{aligned}
& I_{o f f, i}(\mathbf{P}, \alpha) \\
& =\frac{B(1-\alpha) T}{G} \log _{2}\left(1+\frac{G P_{i} h_{i}}{\sum_{n \in \mathcal{N}_{1}, n \neq i}, P_{n} h_{n}+N_{0} B}\right),
\end{aligned}
$$

$\forall i \in \mathcal{N}_{1}$, where $P_{i}$ denotes the transmit power of the $i$-th user. $B$ and $N_{0}$ denote the channel bandwidth and the receiver noise power. $\sum_{n \in \mathcal{N}_{1}, n \neq i}, P_{n} h_{n}$ represents the interference caused by the other offloading users to the $i$-th user due to the imperfect spreading code. Accordingly, the task offloading rate is

$$
r_{o f f, i}(\mathbf{P}, \alpha)=I_{o f f, i} / T, \quad \forall i \in \mathcal{N}_{1} .
$$

As we neglect the edge computation time and result downloading time, $r_{o f f, i}$ equals to the number of processed raw data per second. Under the energy harvesting constrain, the transmit power $P_{i}$ of $i$-th user is limited by $P_{i}(1-\alpha) T \leq E_{i}+e_{i}$, where $e_{i}$ denotes the residual energy left from the previous time frame and is assumed zero for simplicity and without loss of generality in the following analysis. Equivalently, we have

$$
P_{i} \leq \max \left\{\frac{\nu h_{i} \alpha P_{0}}{1-\alpha}, q_{\max }\right\} \triangleq P_{\max , i},
$$

where $q_{\max }$ denotes the maximum transmission power and $P_{\max , i}$ denotes the maximum allowable power of the $i$-th WD due to the energy harvesting constraint (1). Since the value of $h_{i}$ and $e_{i}$ for each WD are different, the corresponding maximum power $P_{\max , i}$ is also different between different users in general.

\section{Local Computing Mode}

Because energy harvesting and task computation use separate circuits at the user device, a mode- 0 user can harvest RF energy and compute its task at the same time, which indicates that it can compute for the entire duration of $T 2$ Let $C_{i}>0$

\footnotetext{
${ }^{2}$ Here, we consider that each WD has sufficient initial energy to perform local computation at the beginning of a time frame (such as in [7]).
} 
denote the number of CPU cycles required to process one bit of raw data, which is assumed equal for all the WDs. Let $f_{i}$ represent the CPU frequency (cycles per second) for processing the task of the $i$-th user. It is shown in [7] that an energy-constrained user should compute throughout the time frame to maximize its computation rate, i.e., the number of information bits processed within a unit time. Accordingly, the energy on computation is constrained as [7]

$$
k_{i} f_{i}^{3} T \leq E_{i}, \forall i \in \mathcal{N}_{0},
$$

where $k_{i}$ denotes the computation energy efficiency coefficient. By exhausting the harvested energy, we have $f_{i}^{*}=\left(\frac{E_{i}}{k_{i} T}\right)^{\frac{1}{3}}$. By substituting (1) to (5), the maximum local computation rate of the $i$-th user is

$$
r_{l o c, i}^{*}(\alpha)=\frac{f_{i}^{*}}{C_{i}}=\frac{\left(\nu P_{0}\right)^{\frac{1}{3}}}{C_{i}}\left(\frac{h_{i}}{k_{i}}\right)^{\frac{1}{3}} \alpha^{\frac{1}{3}}=\eta\left(\frac{h_{i}}{k_{i}}\right)^{\frac{1}{3}} \alpha^{\frac{1}{3}}, i \in \mathcal{N}_{0},
$$

where $\eta=\frac{\left(\nu P_{0}\right)^{\frac{1}{3}}}{C_{i}}$ is a fixed parameter.

\section{Problem Formulation}

In this article, our main purpose is to maximize the weighted sum computation rate of all users in a time frame, which reflects the data processing capability of the considered wireless powered MEC network. Based on the analysis above, the computation rate of each user is related to its computation offloading decisions. Let $w_{i}>0$ represent the positive weight assigned to the $i$-th user. We denote the offloading strategy as $\mathbf{x}=\left\{x_{i}, \forall i \in \mathcal{N}\right\}$, where $x_{i}=1$ denotes that the $i$-th user offloads its computation task and $x_{i}=0$ denotes that it computes the computation task locally. Accordingly, the weighted sum computation rate of the wireless powered MEC network in a time frame is denoted as

$$
F(\mathbf{x}, \mathbf{P}, \alpha)=\sum_{i=1}^{N} w_{i}\left(1-x_{i}\right) r_{l o c, i}^{*}(\alpha)+w_{i} x_{i} r_{o f f, i}(\mathbf{P}, \alpha) .
$$

Mathematically, the rate maximization problem is formulated as follows:

$$
\begin{aligned}
(\mathrm{P} 1): \mathrm{F}^{*}= & \max _{\mathbf{x}, \mathbf{P}, \alpha} F(\mathbf{x}, \mathbf{P}, \alpha) \\
& \text { subject to } \\
& P_{i} \leq \max \left\{\frac{\nu h_{i} \alpha P_{0}}{1-\alpha}, q_{\max }\right\}, \quad \forall i \in \mathcal{N}_{1}, \\
& 0 \leq \alpha \leq 1, x_{i} \in\{0,1\}, i=1, \cdots, N .
\end{aligned}
$$

Problem (P1) is highly non-convex and hard to solve for optimum. Firstly, it contains the combinatorial mode selection binary variables. Secondly, even if the computation modes are given, the remaining multi-user power control in interference channel is a non-convex hard problem. Intuitively, it is not optimal for all users to compute locally or to offload all their tasks for edge execution. On the one hand, by letting all users to offload their tasks will result in severely high interference levels, thus reducing the total task offloading rate. It is therefore important to select a proper set of mode- 1 users to maximize the computation rate. In the following section, we propose an efficient algorithm to optimize (P1).

\section{Proposed Algorithm to (P1)}

We decompose the original problem into two subproblems: computation resource allocation on $\{\alpha, P\}$ for a fixed $\mathbf{x}$ and offloading decision optimization to find the optimal $\mathbf{x}$. In this section, we show how to solve the two subproblems respectively.

\section{A. Optimal Time Allocation and Power Control given $\mathbf{x}$}

To begin with, we assume $\mathbf{x}$ (or equivalently $\mathcal{N}_{0}$ ) and $\alpha$ are given, the problem reduces to the following power control problem

$$
F\left(\mathcal{N}_{0}, \alpha\right)=\max _{\mathbf{P}} F\left(\mathcal{N}_{0}, \alpha, \mathbf{P}\right) .
$$

Notice once $\mathcal{N}_{0}$ and $\alpha$ are given, the computation rate of each mode-0 user are automatically obtained from (6). Besides, the transmit power constraint $P_{\max , i}$ are fixed value for each mode-1 user from (4). Accordingly, we only need to optimize the weighted sum rate of all mode-1 users in (P1) by controlling each user's transmit power under respective maximum power constraint. The objective of (P1) is reduced to

$$
R(\mathbf{P})=\sum_{k \in \mathcal{N}_{1}} w_{k} r_{o f f, k}(\mathbf{P}),
$$

where $\mathbf{P}=\left\{P_{k}, \forall k \in \mathcal{N}_{1}\right\}$ denotes the transmission power vector of mode-1 users. We aim to find the optimal power allocation $\mathbf{P}^{*}$ that maximizes the computation rates of the mode-1 users, which is formulated as

$$
\begin{array}{ll}
\underset{\mathbf{P}}{\operatorname{maximize}} & R(\mathbf{P}) \\
\text { subject to } & P_{i} \leq P_{\max , i}, \quad \forall i \in \mathcal{N}_{1} .
\end{array}
$$

Note that (11) is a non-convex problem, which corresponds to a classic multi-user power control problem in an uplink wireless communication system. In the following subsection, we implement a direct FP approach to find a near-optimal solution. It is shown in [16] that the direct FP approach is effective in control the transmit power of multiple users to maximize the weighted sum throughput performance in the uplink interference channel. For simplicity of illustration, we denote $M=\left|\mathcal{N}_{1}\right|$. We introduce auxiliary variables $y_{i}{ }^{\prime} s$ and apply the quadratic transform technique to each SINR term, which leads to the following reformulation

$$
\begin{array}{cl}
\underset{\mathbf{P}, \mathbf{y}}{\operatorname{maximize}} & f(\mathbf{P}, \mathbf{y}) \\
\text { subject to } & P_{i} \leq P_{\max , i}, \quad \forall i \in \mathcal{N}_{1} \\
& y_{i} \in R, \quad \forall i \in \mathcal{N}_{1}
\end{array}
$$

where the new objective $f$ is

$$
\begin{aligned}
f(\mathbf{P}, \mathbf{y})=\sum_{i=1}^{M} w_{i} \frac{B(1-\alpha) T}{G} \log _{2}\left[1+2 y_{i} \sqrt{G P_{i} h_{i}}\right. \\
\left.-y_{i}{ }^{2}\left(\sum_{n \in \mathcal{N}_{1}, n \neq i,} P_{n} h_{n}+N_{0} B\right)\right] .
\end{aligned}
$$




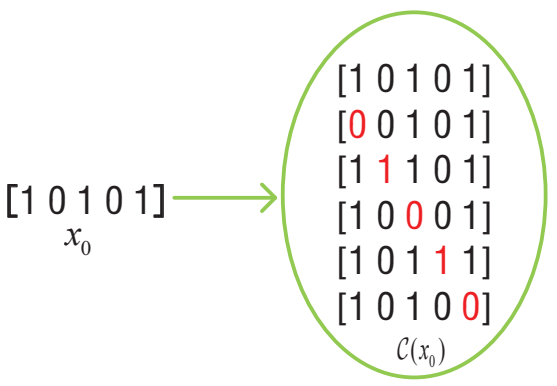

Fig. 2. The sampling sets $\mathcal{C}\left(x_{0}\right)$ of a given mode selection $x_{0}$

When $\mathbf{y}$ is fixed, the quadratic transform (13) is concave in $\mathbf{P}$. Therefore, the optimal $\mathbf{P}$ can be efficiently obtained through conventional convex optimization algorithms, e.g., interior point method.

On the other hand, when $\mathbf{P}$ is held fixed, the optimal $y_{i}$ can be found in closed form as

$$
y_{i}^{*}=\frac{\sqrt{G P_{i} h_{i}}}{\sum_{n \in \mathcal{N}_{1}, n \neq i,} P_{n} h_{n}+N_{0} B}, \forall i \in \mathcal{N}_{1} .
$$

Therefore, we can optimize $y_{i}$ and $P_{i}$ in an iterative fashion as shown in Algorithm 1. Because the objective is nondecreasing in each update of $\mathbf{y}$ or $\mathbf{P}$, the algorithm guarantees to converge asymptotically.

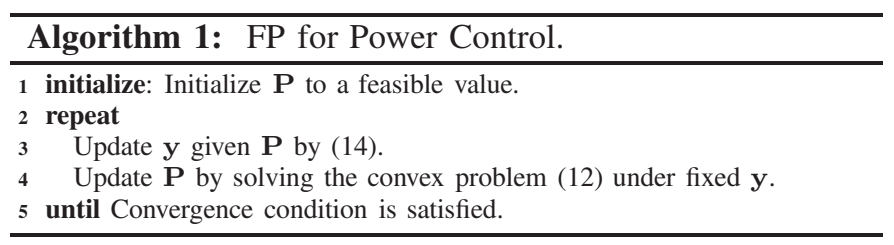

Now that $F\left(\mathcal{N}_{0}, \alpha\right)$ can be efficiently obtained, we can find the optimal time allocation given $\mathcal{N}_{0}$, denoted by

$$
F\left(\mathcal{N}_{0}\right)=\max _{\alpha \in[0,1]} F\left(\mathcal{N}_{0}, \alpha\right)
$$

via a simple one dimension search over $\alpha \in[0,1]$. One efficient method is gold section search [17], which is omitted here for brevity.

\section{B. Stochastic Local Search for Mode Selection Optimization}

The remaining problem is to find the optimal binary computation mode selection vector $\mathbf{x}$. From the above discussions, we denote the achievable rate given a mode selection vector $\mathrm{x}$ as

$$
F(x)=\max _{\alpha} F(x, \alpha)
$$

Here, we apply a Stochastic Local Search algorithm to find the optimal $\mathbf{x}$.

The proposed algorithm iteratively updates $\mathbf{x}$ in a probabilistic manner, while at most one of the $N$ entries of $\mathbf{x}$ is changed in each iteration. We denote the mode selection vector in the $l$-th iteration as $\mathrm{x}^{l}$, where $\mathrm{x}^{0}$ denotes the initial mode selection solution. Then, in the $l$-th iteration, we consider $(N+1)$ candidate solutions, denoted by $\mathcal{C}\left(\mathrm{x}^{l}\right)$, including $\mathrm{x}^{l}$ itself and $N$ neighboring solutions that are off by one bit [4]. As an illustrating example, the candidate solution set of a specific $x_{0}$ is shown in Fig. 2. We select one of the $(N+1)$ candidate solutions as $\mathrm{x}^{l+1}$ in the subsequent iteration. The update is performed according to the following probability

$$
p\left(\mathcal{C}\left(\mathbf{x}^{l}\right)_{i}\right)=\frac{\exp \left(-\beta / F\left(\mathcal{C}\left(\mathbf{x}^{l}\right)_{i}\right)\right)}{\sum_{\mathcal{C}\left(\mathbf{x}^{l}\right)_{i} \in \mathcal{C}\left(\mathbf{x}^{l}\right)} \exp \left(-\beta / F\left(\mathcal{C}\left(\mathbf{x}^{l}\right)_{i}\right)\right)},
$$

$i=1, \cdots, N+1$, where $\mathcal{C}\left(\mathbf{x}^{l}\right)_{i}$ denotes $i$-th feasible solution in candidate solution set $\mathcal{C}\left(\mathbf{x}^{l}\right)$ and $\beta$ denotes a non-negative temperature parameter. Intuitively, $\mathcal{C}\left(\mathbf{x}^{l}\right)_{i}$ that yields a higher $F\left(\mathcal{C}\left(\mathrm{x}^{l}\right)_{i}\right)$ value will be picked with a higher probability, such that (P1) tends to converge to a better solution upon each iteration. Meanwhile, it also has non-zero probability to accept solutions that are worse than the local optimum, thus it is able to escape from the "trap" of of local optimum. Besides, by gradually increasing the temperature parameter $\beta$ as the iterations proceed, we can balance the chance of exploitation (large $\beta$ ) and exploration (small $\beta$ ) over time to converge to an optimum. The overall algorithm to solve (P1) is summarized in Algorithm 2.

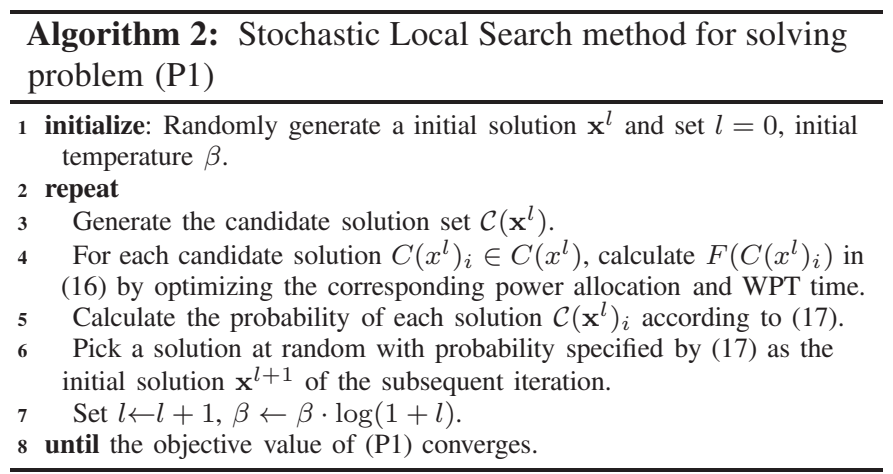

\section{Simulation Results}

In this section, we evaluate the performance of the proposed algorithm using simulations. In all simulations, we use the parameters of Powercast TX91501-3W transmitter with $P_{0}=$ $3 \mathrm{~W}$ (Watt) as the energy transmitter at the HAP and those of P2110 Power harvester as the energy receiver at each WD with $\nu=0.51$ energy harvesting efficiency. Without loss of generality, it is assumed that the noise power is set $N_{0}=-140$ $\mathrm{dBm}$ for all receivers, $q_{\max }=1 \mathrm{~mW}$, and $T=1$. The wireless channel gain $h_{i}$ between any two nodes, either HAP or a WD, follows the free-space path loss model

$$
h_{i}=G_{A}\left(\frac{3 \times 10^{8}}{4 \pi D_{i} f_{c}}\right)^{\lambda}, i=1, \ldots N,
$$

where $D_{i}$ denotes the distance between the HAP and $i$-th $\mathrm{WD}, G_{A}=4.11$ denotes the antenna power gain, $\lambda=2.8$ denotes the path-loss exponent, and $f_{c}=915 \mathrm{MHz}$ denotes the carrier frequency. Besides, we set equal computing efficiency parameter $k_{i}=10^{-26}, i=1, \ldots, N$, and $C_{i}=100$ for all the WDs [7]. For the data offloading mode, the bandwidth $B=10 \mathrm{MHz}$ and the processing gain of the system $G=128$. 

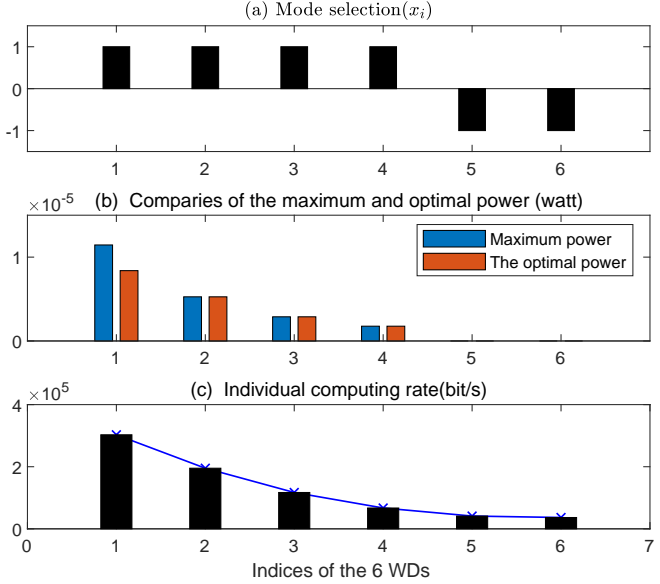

Fig. 3. The figure shows the optimal computing mode solution, the comparison between the maximum power and the optimal power, and individual computing rates.

Without loss of generality, we assume zero residual energy of the previous time frame $e_{i}=0, i=1, \ldots, N$.

In Fig. 3, we first study some properties of the optimal solution to (P1), where we assume a homogeneous special case with $w_{i}=1$ for all the WDs. For simplicity of illustration, we consider $N=6$ and $D_{i}=3+(i-1)$ meters, $i=1, \ldots, 6$. Clearly, the wireless channel of $\mathrm{WD}_{1}\left(D_{1}=3\right)$ that is closest to the AP is the strongest, while the furthest $\mathrm{WD}_{6}\left(D_{6}=8\right)$ has the weakest channel. In all the sub-figures, the $\mathrm{x}$-axis denotes the indices of the 6 WDs. The figure above Fig. 3(a) shows the the optimal mode selection, where $x_{i}=1$ denotes the $i$-th WD selects mode 1 and $x_{i}=-1$ denotes the $i$-th WD selects mode 0 . From the figure above, we can observe that the 4 WDs with stronger wireless channels offload their tasks for edge execution while other two weak WDs perform computations locally. Besides, we plot in Fig. 3(b) the maximum allowable transmission power of a single user and the optimal transmission power under power control. $\mathrm{WD}_{1}$ has the highest maximum transmission power because it harvests the most energy for information transmission. Meanwhile, the 4 users that work in mode- 1 do not always transmit at the maximum power. Specifically, $\mathrm{WD}_{1}$ transmits at a lower power than the maximum allowable power, while other three users 2-4 transmit with the maximum power. Intuitively, the one with the strongest WD-to-HAP channel $\left(\mathrm{WD}_{1}\right)$ may cause strong interference to other users if transmitting at full capacity. From Fig. 3(c), we further observe that mobile edge computing improves the computation rate of WDs effectively. For example, the computing rate of the mode- $0 \mathrm{WD}_{5}$ is merely $12.8 \%$ higher than another mode- $0 \mathrm{WD}_{6}$, while the computing rate of the mode- $1 \mathrm{WD}_{4}$ is $63.1 \%$ higher than $\mathrm{WD}_{5}$.

In Fig. 4, we evaluate the impact of path loss exponent to the optimal mode selections. The three figures show the number of mode-1 WDs decreases when the path loss exponent $\lambda$ increases. This is because the larger channel attenuation under larger $\lambda$ leads to higher cost on task data offloading.

In Fig. 5 and 6, we evaluate the computation performance of the proposed optimization methods. In particular, we con-

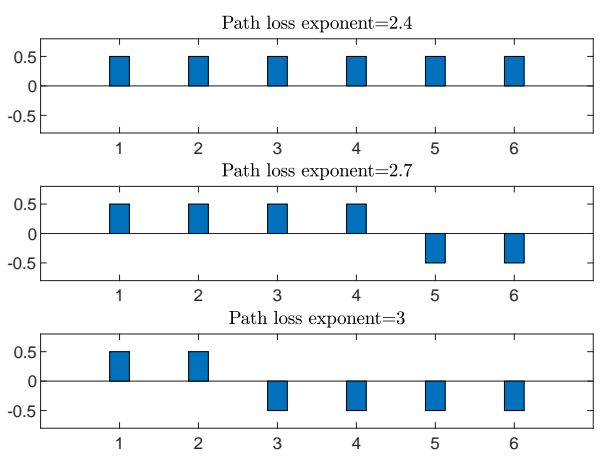

Fig. 4. Optimal mode selections when path loss exponent varies.

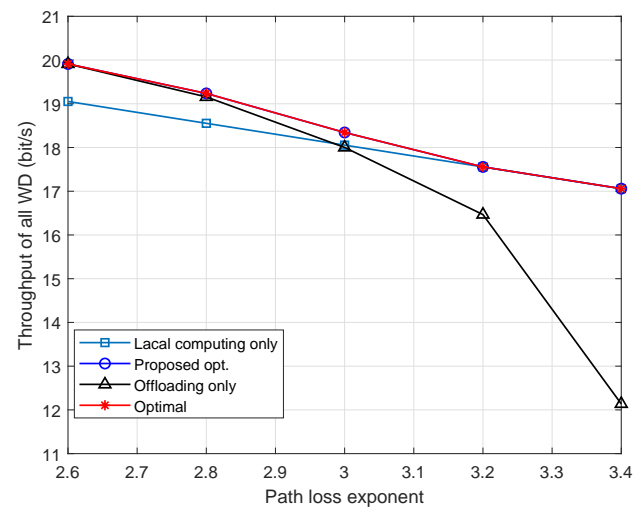

Fig. 5. Computation rate performance of different schemes when path loss exponent varies.

sider three representative benchmark methods for performance comparisons:

1) Offloading only: all the WDs offload their tasks to the AP for remote execution;

2) Local computing only: all the WDs compute their tasks locally;

3) Optimal: search all the $2^{N}$ combinations of computing mode selections and output the one with the best performance.

In Fig. 5, we compare computation performance of different schemes when path loss exponent $\lambda$ increases from 2.6 to 3.4. The network setups, e.g., the number of WDs, WD locations and weights, are the same as those used in Fig. 3. We see that the performance of all the considered schemes degrade as $\lambda$ increases (i.e., channels become weaker). Specifically, when wireless channels are strong (that is, when path loss exponent $\lambda$ is small) the offloading only scheme achieves near optimal performance. However, as $\lambda$ increase, the performance of the offloading only scheme quickly degrades, because the offloading rates severely suffer from the weak channels. On the other hand, when $\lambda$ is large (weak channels), the all local computing scheme is optimal. The proposed optimization method achieves identical performance as the optimal scheme and significantly outperforms the other three benchmark methods.

In Fig. 6, we compare the computation performance of 


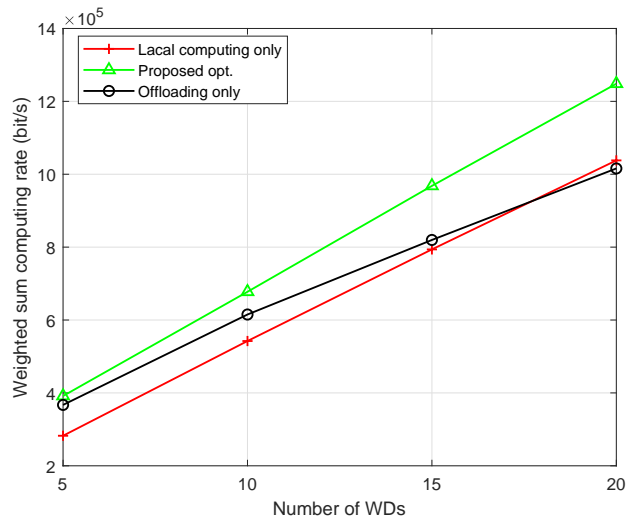

Fig. 6. When the number of users changes, the performance of different schemes is compared.

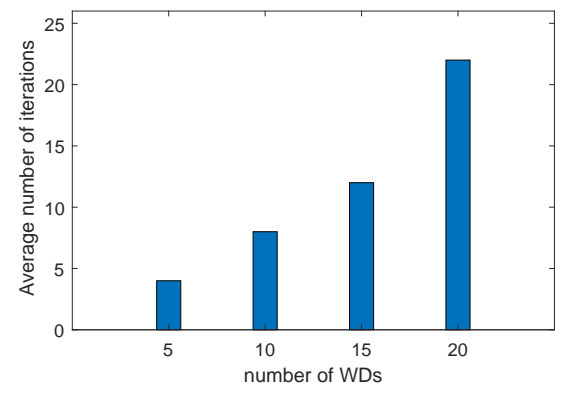

Fig. 7. Average number of Stochastic Local Search algorithm search times when the number of WDs varies.

different methods when the number of WDs $N$ varies from 5 to 20 . For each $\mathrm{WD}_{i}$, the distance to $\mathrm{AP}$ is uniformly generated as $D_{i} \sim U(2.5,10)$, and they has homogeneous weight $w_{i}=1$. Each point is an average performance of 20 independent device placement. The optimal performance is not plotted in Fig. 6, because the mode-enumeration based optimal method is computationally infeasible when $N$ is larger, e.g., $N=20$. We compare the computation performance of the proposed optimization method with that of the other two benchmark methods. We can see that in all the schemes, the sum rates increase with the number of WDs. The proposed optimization method significantly outperforms the other two benchmark methods. In particular, the average computation rate of our proposed optimization method is about $90 \%$ and $20 \%$ higher than the local-computing-only scheme and offloading-only scheme, respectively.

In Fig. 7, we characterize the computation complexity of the proposed Stochastic Local Search algorithm. We plot the average number of searches when the number of WDs $N$ varies from 5 to 20 . Here, we consider the algorithm converges if the difference of objective value between two adjacent iterations is less than $10^{-4}$. We see that average number of Stochastic Local Search algorithm iterations increases almost linearly with the number of WDs. The results indicate that Stochastic Local Search algorithm can quickly converge to the global optimal solutions and is applicable to large-size networks.

\section{CONCLUSIONS}

In this paper, we studied the resource allocation problem of a multi-user wireless powered MEC system using DSCDMA to coordinate the task offloading of the users. We formulated the computation rate maximization problem as a joint optimization of individual computing mode selection, transmission power control and WPT time allocation. To tackle the problem, we first assumed that the computation mode selection is given and applied a direct FP approach to solve the rate maximization problem in the uplink interference channel. On top of that, a simple linear search method is applied to find the optimal WPT time. In addition, we further proposed a Stochastic Local Search algorithm to optimize the individual computing mode selections. By comparing with representative benchmark methods, we showed that the proposed the scheme can significantly improve the computation performance of the wireless powered MEC system based on DS-CDMA.

\section{REFERENCES}

[1] Y. Mao, C. You, J. Zhang, K. Huang, and K. B. Letaief, "A survey on mobile edge computing: the communication perspective," IEEE Commun Surveys Tuts., vol. 19, no. 4, pp. 2322-2358, Aug. 2017.

[2] Y. Mao, J. Zhang, and K. B. Letaief, "Dynamic computation offloading for mobile-edge computing with energy harvesting devices", IEEE J. Sel. Areas Commun., vol. 34, no. 12, pp. 3590-3605, Dec. 2016.

[3] M. H. Chen, B. Liang, and M. Dong, "Joint offloading decision and resource allocation for multi-user multi-task mobile cloud", Proc. IEEE Int. Conf. Commun. (ICC), pp. 1-6, May. 2016.

[4] J. Yan, S. Bi, and Y. J. Zhang, "Optimal offloading and resource allocation in mobile-edge computing with inter-user task dependency," Proc. IEEE GLOBECOM, Dec. 2018.

[5] X. Lu, P. Wang, D. Niyato, D. I. Kim, and Z. Han, "Wireless networks with RF energy harvesting: a contemporary survey," IEEE Commun. Surveys Tuts., vol. 17, no. 2, pp. 757-789, 2015.

[6] S. Bi, Y. Zeng, and R. Zhang, "Wireless powered communication networks: an overview," IEEE Wireless Commun., vol. 23, no. 2, pp. 10-18, Apr. 2016.

[7] S. Bi and Y. J. Zhang, "Computation rate maximization for wireless powered mobile-edge computing with binary computation offloading," IEEE Trans. Wireless Commun., vol. 17, no. 6, pp. 4177-4190, Jun. 2018.

[8] L. Huang, S. Bi, and Y. J. Zhang, "Deep reinforcement learning for online computation offloading in wireless powered mobile-edge computing networks," IEEE Trans. Mobile Compt., DOI: 10.1109/TMC2019.2928811, Jul. 2019.

[9] F. Wang, J. Xu, X. Wang, and S. Cui, "Joint offloading and computing optimization in wireless powered mobile-edge computing systems," IEEE Trans. Commun., vol. 17, no. 3, pp. 1784-1797, Mar. 2018.

[10] H. Q. Le, H. Al-Shatri, and A. Klein, "Efficient resource allocation in mobile-edge computation offloading: completion time minimization,' 2017 IEEE ISIT, pp. 2513-2517, Jun. 2017.

[11] C. You, K. Huang, H. Chae, and B. H. Kim, "Energy-efficient resource allocation for mobile-edge computation offloading," IEEE Trans. Wireless Commun., vol. 16, no. 3, pp. 1397-1411, Mar. 2017.

[12] F. Wang, J. Xu, and Zhi. Ding, "Multi-antenna NOMA for computation offloading in multiuser mobile edge computing systems," IEEE Trans. Commun., vol. 67, no. 3, Mar. 2019.

[13] L. Zhang, Y. C. Liang, and M. Xiao, "Spectrum sharing for Internet of Things: a survey," IEEE Wireless Commun., vol. 26, no. 3, Dec. 2018.

[14] D. Tse, and P. Viswanath, "Fundamentals of wireless communications," in Cambridge University Press, 2005.

[15] J. M. Kang, I. M. Kim, and D. I. Kim, "Joint Tx power allocation and Rx power splitting for SWIPT system with multiple nonlinear energy harvesting circuit," IEEE Wireless Commun. Lett., vol. 8, no. 1, pp. 5356, Feb. 2019.

[16] C. S. Chen, K. Shum, and C. W. Sung, "Fractional Programming for Communication SystemsłPart I: Power Control and Beamforming," IEEE Trans. on signal pro. , vol. 66, no. 10, May. 2018.

[17] T. Scherrer, S. Y. Kim, and C. Yi, "Low complexity, real-time adjusted power management policy using golden section search," in Int. SoC Des. (ISOCC), nov. 2013, 229-232. 